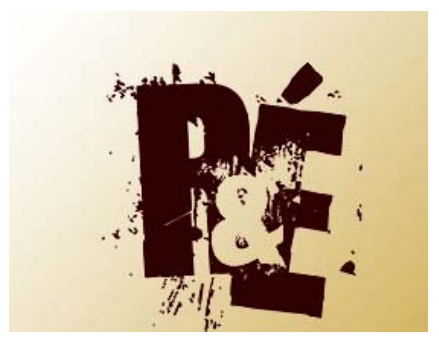

Appel à communication

\section{Revue « Recherches et Educations » publication prévue en 2012}

Site internet de la revue : http://rechercheseducations.revues.org/

Thématique du numéro :

\title{
La construction d'une professionnalité en éducation : entre accompagnement et reconnaissance professionnelle ?
}

Il s'agira dans ce numéro d'étudier les démarches et processus d'accompagnement des professeurs stagiaires en formation ou lors de leur première année d'exercice en liens avec la reconnaissance professionnelle. De façon complémentaire, de caractériser le type d'interaction construite entre les interlocuteurs et de préciser les objets de la reconnaissance d'une professionnalité en cours de construction. Les modalités à l'œuvre comme par exemple les analyses, les évaluations conduites lors de l'entretien professionnel, les démarches de portfolio seront étudiées.

Ce numéro vise à clarifier le processus d'accompagnement au regard du processus de reconnaissance professionnelle dans la rencontre entre un expert et un novice. Il cherche en particulier à mettre en évidence les liens entre l'accompagnement et la reconnaissance professionnelle, moteurs d'une valorisation d'entrée dans le métier. En effet, le choc de la réalité du terrain demande au novice un engagement professionnel auquel il n'est pas préparé. Le processus d'accompagnement couplé à une démarche de reconnaissance nous paraît être une démarche professionnelle prometteuse pour permettre les premiers pas sur le terrain professionnel. La préparation des stages constitue certes une approche, il nous semble que le suivi qui est pensé en terme de valorisation du professionnel se construisant peut contribuer à la demande d'appui des novices. Ces derniers pouvant alors faire face aux exigences du terrain professionnel.

Les remarques des professionnels sur l'absence de reconnaissance du travail effectué traduisent le manque de retours positifs. L'apprêt technique de certaines rencontres ne peut endiguer ce sentiment de non reconnaissance. Aussi, est-ce la raison de ce numéro que de questionner l'importance des transactions de reconnaissance dans un contexte de professionnalisation. Les contributeurs se situeront dans une perspective de mise en évidence de ce qui se fait quand bien même des régulations de l'activité deviennent nécessaires. Ils expliciteront les démarches qui permettent aussi bien la valorisation que la régulation de l'activité.

Lectorat potentiel :

Les formateurs, les conseillers pédagogiques, les cadres du système éducatifs : chefs d'établissement, inspecteurs

Echéancier :

Propositions à adresser à Anne Jorro : jorro@ univ-tlse2.fr pour le 15 janvier 2011. Un résumé de 1000 signes maximum est attendu. 